07;08;09

\title{
Влияние саморазогрева на модуляционные характеристики микродискового лазера
}

\author{
(c) А.Е. Жуков ${ }^{1}$, Э.И. Моисеев ${ }^{1,2}$, А.М. Надточий ${ }^{1,2}$, Н.В. Крыжановская ${ }^{1}$, М.М. Кулагина ${ }^{3}$, С.А. Минтаиров ${ }^{3}$, \\ Н.А. Калюжный ${ }^{3}$ Ф.И. Зубов ${ }^{2}$, М.В. Максимов ${ }^{2}$ \\ ${ }^{1}$ Национальный исследовательский университет „Высшая школа экономики“, Санкт-Петербург, Россия \\ ${ }^{2}$ Санкт-Петербургский национальный исследовательский Академический университет им. Ж.И. Алфёрова РАН, \\ Санкт-Петербург, Россия \\ ${ }^{3}$ Физико-технический институт им. А.Ф. Иофрфе РАН, Санкт-Петербург, Россия \\ E-mail: zhukale@gmail.com
}

Поступило в Редакцию 2 марта 2020 г.

В окончательной редакции 2 марта 2020 г.

Принято к публикации 3 марта 2020 г.

\begin{abstract}
Экспериментально исследовано быстродействие микродисковых лазеров с квантовыми точками, работающих при комнатной температуре без термостабилизации, а также выполнен расчет наибольшей полосы модуляции микродисков различного диаметра. Показано, что учет эффекта саморазогрева микролазера при больших токах смещения, проявляющегося в уменьшении предельного быстродействия и увеличении тока, при котором достигается максимальная полоса модуляции, позволяет хорошо описать экспериментальные данные. Наибольшее влияние саморазогрев оказывает на микролазеры малого диаметра (менее $20 \mu \mathrm{m}$ ).
\end{abstract}

Ключевые слова: высокочастотная модуляция, микролазер, полупроводниковый лазер, квантовые точки.

DOI: 10.21883/PJTF.2020.11.49488.18271

В последние годы значительное внимание уделяется микролазерам на основе микродисковых и микрокольцевых резонаторов с активной областью на основе квантовых точек, что обусловлено возможностью достижения малых размеров прибора (вплоть до $1 \mu \mathrm{m}$ при оптической накачке и менее $10 \mu \mathrm{m}$ при инжекционной накачке [1]) и низких пороговых токов $\left(250 \mathrm{~A} / \mathrm{cm}^{2}\right.$ при комнатной температуре [2]) в сочетании с простотой изготовления таких микролазеров. Для их создания нет необходимости применять распределенные брэгговские отражатели, токовые апертуры, многоэтапную литографию, а используемые эпитаксиальные гетероструктуры аналогичны применяемым при изготовлении полосковых лазеров. Одним из основных предполагаемых приложений микродисковых лазеров является оптическая передача данных на сверхкороткие расстояния, в предельном случае внутри оптоэлектронной интегральной схемы, в том числе на основе кремния. В связи с этим одной из наиболее важных приборных характеристик микродискового лазера является полоса модуляции $f_{3 \mathrm{~dB}}$, определяемая как частота, при которой эффективность прямой модуляции спадает относительно ее низкочастотного значения на $3 \mathrm{~dB}$.

Ограничение частоты модуляции может быть обусловлено множеством факторов [3], одним из которых является увеличение температуры прибора при протекании электрического тока большой плотности. Явление саморазогрева наиболее характерно для лазеров с малой площадью протекания тока и в связи с этим активно исследовалось применительно к поверхностно-излучающим лазерам с вертикальным резонатором [4,5]. В то же время влияние саморазогрева на высокоча- стотные характеристики микродисковых лазеров [6,7] ранее, насколько нам известно, не изучалось. В настоящей работе с помощью сравнения экспериментальных данных и результатов численного моделирования исследуется относительный вклад саморазогрева в ограничение предельной частоты модуляции инжекционных микродисковых лазеров с квантовыми точками, работающих при комнатной температуре без принудительного охлаждения.

Приведенные в настоящей работе экспериментальные значения полосы модуляции $f_{3 \mathrm{~dB}}$ определялись на основе малосигнальной амплитудно-частотной характеристики $A(f)$, измеряемой в диапазоне $0.1-20 \mathrm{GHz}$ при различных токах смещения. Мы анализируем результаты, полученные при исследовании микролазеров с квантовыми точками (In, Ga)As высокой плотности [8]. Микролазеры формировались при глубоком травлении эпитаксиальной гетероструктуры с последующим изготовлением электрических контактов к подложке и верху цилиндрической мезы. Такие микролазеры продемонстрировали на данный момент наибольшие значения полосы модуляции более $6 \mathrm{GHz}$ [9], что позволило реализовать с их помощью оптическую передачу данных со скоростью $10 \mathrm{~Gb} / \mathrm{s}$ [10].

Использованные в расчетах параметры микролазеров приведены в таблице. Пороговый ток исследуемых микродисковых лазеров характеризуется двухкомпонентной зависимостью от диаметра микролазера: слагаемое, пропорциональное площади прибора, может быть сопоставлено с рекомбинацией в объеме активной области, а слагаемое, пропорциональное периметру, с поверхностной рекомбинацией на боковых стенках. 
Параметры микродисковых лазеров, использованные при расчетах

\begin{tabular}{|c|c|c|}
\hline Параметр & Значение & Лит. ссылка \\
\hline $\begin{array}{l}\text { Диаметр микродиска } d \\
\text { Напряжение открывания диода } U_{0} \\
\text { Удельное электрическое сопротивление } \rho_{S} \\
\text { Удельное тепловое сопротивление } \rho_{T} \\
\text { Удельный пороговый ток: } \\
\quad \text { компонента рекомбинации в объеме } J_{2} \\
\quad \text { компонента поверхностной рекомбинации } J_{1} \\
\text { Характеристическая температура } T_{0} \\
K \text {-фактор } \\
\text { Смещение коэффициента затухания релаксационных колебаний } \gamma_{0} \\
\text { Коэффициент эффективности модуляции МСЕF } \\
\text { Паразитная частота отсечки } f_{C} \\
\text { Коэффициент нелинейного насыщения усиления } \varepsilon_{P}\end{array}$ & $\begin{array}{c}10-30 \mu \mathrm{m} \\
1.446 \mathrm{~V} \\
1 \cdot 10^{-4} \Omega \cdot \mathrm{cm}^{2} \\
2.8 \cdot 10^{-3} \mathrm{~cm}^{2} \cdot \mathrm{K} / \mathrm{W} \\
\\
630 \mathrm{~A} / \mathrm{cm}^{2} \\
0.75 \mathrm{~A} / \mathrm{cm} \\
90 \mathrm{~K} \\
0.8 \mathrm{~ns} \\
1.2 \mathrm{~ns}+40 \mathrm{GHz} \cdot \mu \mathrm{m} / d \\
40\left(\mathrm{GHz} / \mathrm{mA}^{0.5}\right) \cdot \mu \mathrm{m} / d \\
5 \mathrm{GHz} \\
<0.1 \mathrm{~mW}-1\end{array}$ & $\begin{array}{c}{[9]} \\
{[12]} \\
{[13]} \\
{[13]} \\
{[9]} \\
\text { Наст. paб. } \\
{[14]}\end{array}$ \\
\hline
\end{tabular}

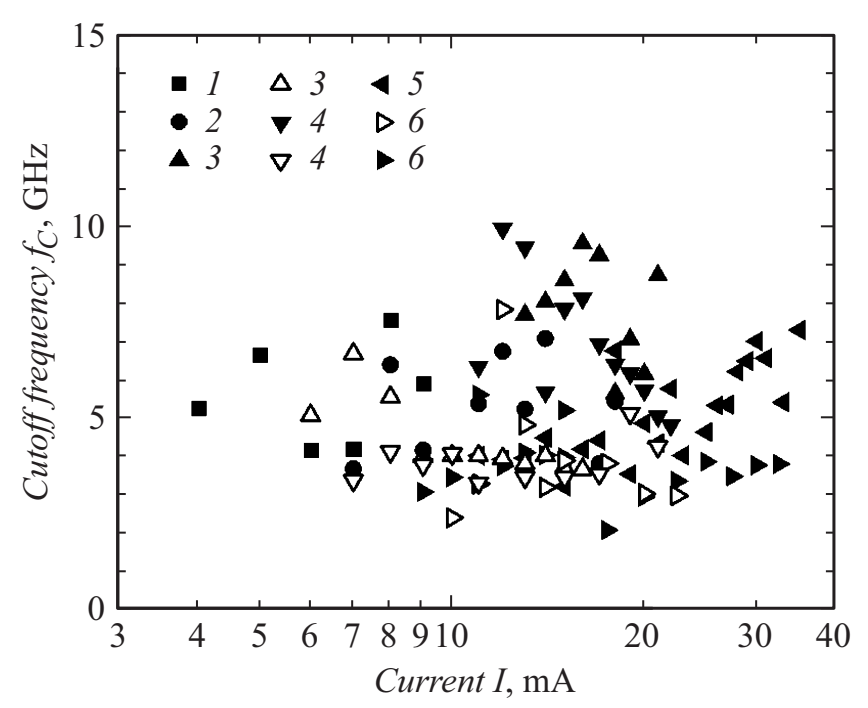

Рис. 1. Значения паразитной частоты отсечки, экспериментально определенные для микродисковых лазеров разного диаметра при различном токе смещения. Диаметр микродиска, $\mu \mathrm{m}: 1-10,2-14,3-19,4-23,5-27,6-31$.

$K$-фактор не демонстрирует регулярной зависимости от диаметра микролазера, что согласуется с предсказаниями теории [15], согласно которым зависящие от диаметра радиационные потери, обусловленные кривизной резонатора, становятся заметными, лишь когда его размер сопоставим с длиной волны излучения. Коэффициент нелинейного насыщения усиления пренебрежимо мал, что обусловлено низкой оптической мощностью микродисковых лазеров.

Паразитная частота отсечки $f_{C}$, связанная с емкостными и/или индукционными элементами СВЧ-схемы, а также с конечными временами транспорта и захвата носителей в активную область лазера, была определена подгонкой экспериментальной модуляционной характеристики $A(f)$ с помощью выражения, которое было получено из анализа скоростных уравнений лазера [16]:

$$
A(f)=\frac{f_{R}^{4}}{\left(f_{R}^{2}-f^{2}\right)^{2}+f^{2}\left(\frac{\gamma}{2 \pi}\right)^{2}} \frac{1}{1+\frac{f^{2}}{f_{C}^{2}}},
$$

где $f_{R}$ - частота релаксационных колебаний, $\gamma$ коэффициент затухания релаксационных колебаний. Рис. 1 обобщает значения $f_{C}$ в зависимости от тока смещения $I$ для микролазеров различного диаметра. Как видно, $f_{C}$ не демонстрирует какой-либо закономерной зависимости от тока и/или диаметра. Среднее значение, использованное при расчетах в настоящей работе, составляет $5 \pm 2 \mathrm{GHz}$.

Ширина полосы модуляции микролазера при некотором токе смещения определялась решением уравнения $A\left(f_{3 \mathrm{~dB}}\right)=1 / 2$. При этом для нахождения зависящих от тока значений частоты релаксационных колебаний и коэффициента их затухания мы использовали известные выражения [17]:

$$
f_{R}=D \sqrt{I-I_{t h}}, \quad \gamma=K f_{R}^{2}+\gamma_{0},
$$

где так называемый $D$-фактор связан в области малых токов смещения с эффективностью модуляции соотношением $\mathrm{MCEF}=\sqrt{1+\sqrt{2}} D, I_{t h}-$ пороговый ток микролазера.

Чтобы учесть явление саморазогрева, мы полагали, что $I_{t h}=I_{0} \exp \left(\Delta T / T_{0}\right), \Delta T=R_{T} U I$, где $I_{0}$ - зависящий от диаметра микролазера пороговый ток в отсутствие саморазогрева (при фиксированной температуре $\left.T_{r e f}=293 \mathrm{~K}\right), \Delta T$ - дополнительное увеличение температуры вследствие выделения джоулева тепла $U I$, $R_{T}$ - тепловое сопротивление, $U=U_{0}+I R_{S}$ - падение напряжения на лазерном диоде, $R_{S}$ - последовательное электрическое сопротивление. Поскольку $D$-фактор пропорционален корню квадратному от дифференциального усиления активной области [17], чтобы учесть снижение дифференциального усиления с ростом температуры, мы полагали $\mathrm{MCEF} \propto \sqrt{T_{r e f} /\left(T_{r e f}+\Delta T\right)}$. Увеличение 

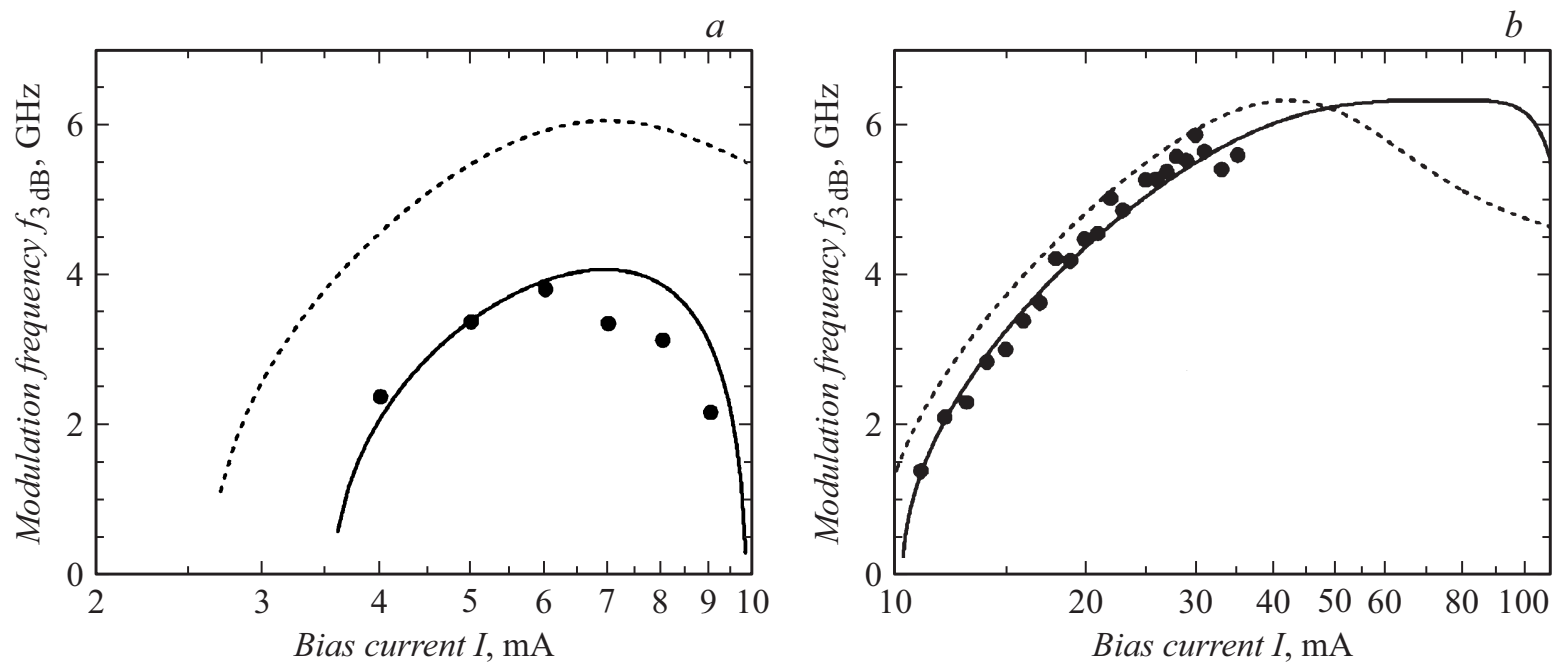

Рис. 2. Зависимость предельной частоты модуляции по уровню $-3 \mathrm{~dB}$ от тока смещения для микродисковых лазеров диаметром $10(a)$ и $27 \mu \mathrm{m}(b)$. Символы - эксперимент, пунктирная линия - расчет без учета саморазогрева, сплошная линия расчет с учетом саморазогрева.
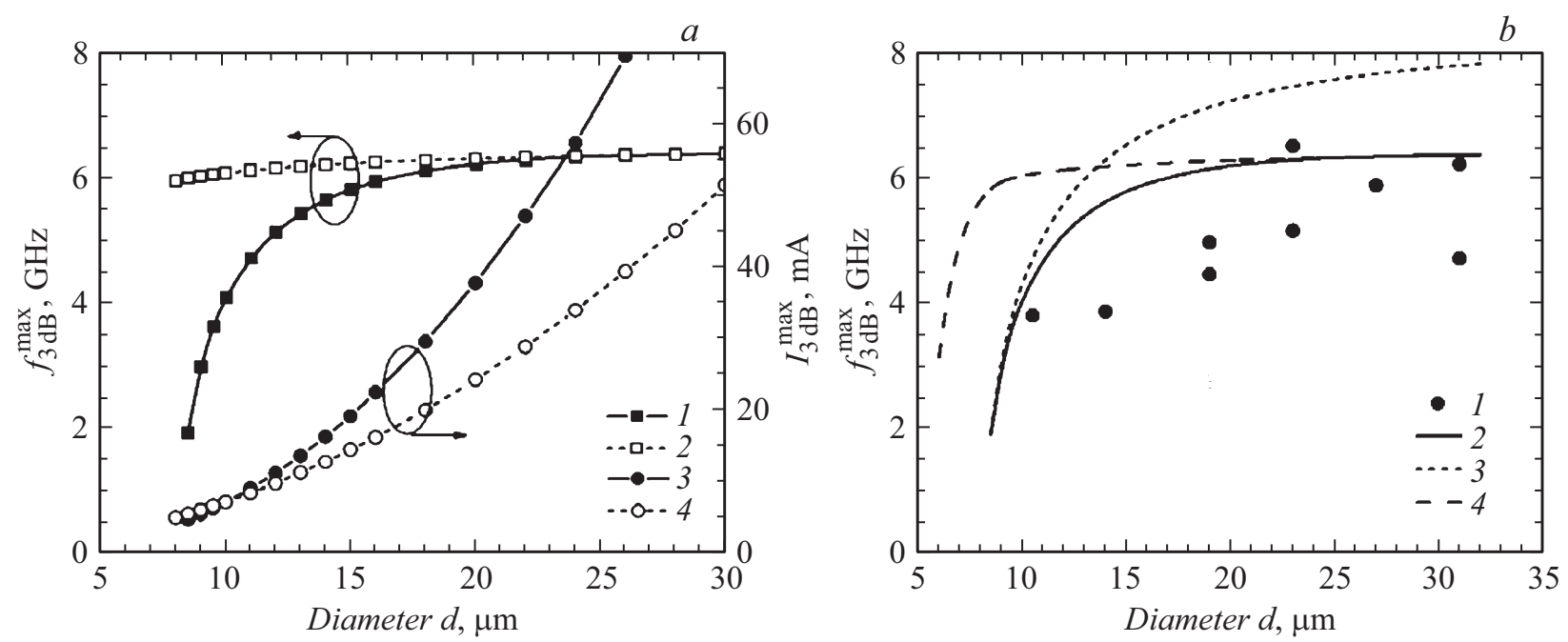

Рис. 3. Зависимости от диаметра микродиска: $a$ - максимальной достижимой частоты модуляции $(1,2)$ и соответствующего ей тока смещения $(3,4)$, рассчитанные с учетом $(1,3)$ и без учета $(2,4)$ саморазогрева; $b$ - максимальной достижимой частоты модуляции ( 1 - эксперимент, 2 - расчет с учетом саморазогрева, 3 и 4 - расчет в предположении в 2 раза большей паразитной частоты отсечки или в 2 раза меньшего теплового сопротивления соответственно).

температуры активной области при больших токах смещения и вызванный этим быстрый рост порогового тока ведут к уменьшению величины $I-I_{t h}$, что может привести к дополнительному (вызванному саморазогревом) снижению эффективности модуляции.

На рис. 2 показаны экспериментальные значения полосы модуляции $f_{3 \mathrm{~dB}}$ в зависимости от тока смещения $I$ для двух микродисковых лазеров относительно малого и относительно большого размера (диаметр $d=10$ и $27 \mu \mathrm{m}$ соответственно). После начального участка возрастания $f_{3 \mathrm{~dB}}$ наблюдается насыщение (для микродиска большего диаметра) и последующий спад (для микродиска меньшего диаметра) предельной частоты модуляции с ростом тока. На рис. 2 приведено также сравнение экспериментальных данных с результатами моделирования, выполненного с учетом и без учета влияния саморазогрева. Как видно, учет саморазогрева позволяет достичь (без использования каких-либо дополнительных подгоночных параметров) хорошего согласия между экспериментом и расчетом для микродисков как большого, так и малого размера. Наиболее существенным учет перегрева оказывается для микролазеров малого диаметра, для которых расчет, выполненный без учета влияния саморазогрева, существенно (примерно на $2 \mathrm{GHz}$ ) завышает значение $f_{3 \mathrm{~dB}}$. Для микродиска большего размера это различие не превышает $0.5 \mathrm{GHz}$.

Для микролазера некоторого диаметра существует наибольшее значение полосы модуляции $f_{3 \mathrm{~dB}}^{\max }$, которое 
достигается при некотором оптимальном токе смещения $I_{3 \mathrm{~dB}}^{\max }$ В том случае, если влияние паразитной отсечки и саморазогрева пренебрежимо мало, лазер достигает наибольшего быстродействия при переходе из режима слабого демпфирования в режим сильного демпфирования релаксационных колебаний. При этом $f_{3 \mathrm{~dB}}^{\max } \approx 2 \sqrt{2} \pi / K$, что для рассматриваемых микролазеров соответствует частоте свыше $10 \mathrm{GHz}$. Экспериментальные и расчетные значения полосы модуляции оказываются заметно ниже. Следует отметить, что расчет зависимости полосы модуляции от тока с учетом саморазогрева для большого микродиска предсказывает наличие плоского максимума, так что $f_{3 \mathrm{~dB}}$ перестает меняться в широком диапазоне токов смещения (в рассмотренном примере от 40 до $100 \mathrm{~mA})$. При этом ток $I_{3 \mathrm{~dB}}^{\max }$ заметно сдвинут в область больших значений по сравнению с положением максимума зависимости без учета перегрева.

Рис. 3, $a$ обобщает расчетные значения $f_{3 \mathrm{~dB}}^{\max }$ и $I_{3 \mathrm{~dB}}^{\max }$ для микролазеров различного диаметра. Если саморазогрев микролазера не принимается во внимание, $f_{3 \mathrm{~dB}}^{\max }$ практически не зависит от размеров прибора, оставаясь на уровне 6-6.5 GHz. Такое поведение связано с тем, что внутренние параметры ( $K$-фактор и паразитная частота отсечки), в основном определяющие быстродействие в отсутствие саморазогрева, не зависят от диаметра. Саморазогрев практически не сказывается на предельном быстродействии больших микродисков, однако ведет к быстрому снижению $f_{3 \mathrm{~dB}}^{\max }$ при уменьшении диаметра менее $20 \mu \mathrm{m}$.

На рис. 3, $b$ символами 1 представлены наибольшие измеренные значения полосы модуляции. Они, как и ожидалось, покрывают область значений, лежащую несколько ниже $f_{3 \mathrm{~dB}}^{\max }$, вычисленной с учетом саморазогрева (сплошная линия 2). В эксперименте также наблюдается заметное уменьшение быстродействия при снижении размеров микродиска. На рисунке приведены также результаты расчетов для двух гипотетических случаев: увеличение паразитной частоты отсечки в 2 раза (пунктирная линия 3) либо двукратное уменьшение теплового сопротивления (штриховая линия 4) по сравнению со значениями соответствующих параметров, приведенными в таблице. Как видно, рост $f_{C}$ оказывает некоторое влияние на быстродействие больших $(d>15 \mu \mathrm{m})$ микродисков, однако практически не влияет на полосу модуляции микролазеров малого диаметра. В противоположность этому улучшение теплового сопротивления ведет к тому, что предельные значения полосы модуляции в микродисках малого диаметра заметно возрастают и оказываются сопоставимыми с $f_{3 \mathrm{~dB}}^{\max }$, а резкий спад $f_{3 \mathrm{~dB}}^{\max }$ наблюдается только для лазеров, диаметр которых меньше $\sim 7 \mu \mathrm{m}$.

Таким образом, предельное быстродействие микродисковых лазеров относительно малого размера определяется в основном явлениями, вызванными перегревом активной области, тогда как для микродисков большего размера максимальная полоса модуляции ограничивается демпфированием релаксационных колебаний и паразитной частотой отсечки. Граница, разделяющая две различные модели поведения при реализованных на практике значениях внутренних параметров микролазеров, соответствует диаметру около $20 \mu \mathrm{m}$. Выше этого размера микродиска предельная частота модуляции практически не зависит от диаметра микродиска, а ниже заметно уменьшается.

\section{Финансирование работы}

Работа выполнена при поддержке Российского научного фонда (соглашение 19-72-30010). Оптические исследования осуществлены в рамках Программы фундаментальных исследований НИУ ВШЭ в 2020 году.

\section{Конфликт интересов}

Авторы заявляют, что у них нет конфликта интересов.

\section{Список литературы}

[1] Mao M.-H., Chien H.-C., Hong J.-Z., Cheng C.-Y. // Opt. Express. 2011. V. 19. N 15. P. 14145-14151.

[2] Moiseev E.I., Kryzhanovskaya N.V., Zubov F.I., Mikhailovskii M.S., Abramov A.N., Maximov M.V., Kulagina M.M., Guseva Yu.A., Livshits D.A., Zhukov A.E. // Semiconductors. 2019. V. 53. N 14. P. 1888-1890.

[3] Vertical-cavity surface-emitting laser devices / Eds H. Li, K. Iga. Heidelberg: Springer-Verlag, 2003. 386 p.

[4] Al-Omari A.N., Lear K.L. // IEEE Photon. Technol. Lett. 2005. V. 17. N 9. P. 1767-1769.

[5] Baveja P.P., Kogel B., Westbergh P., Gustavsson J.S., Haglund A., Maywar D.N., Agrawal G.P., Larsson A. // Opt. Express. 2011. V. 19. N 16. P. 15490-15505.

[6] Lv X.M., Huang Y.Z., Zou L.X., Long H., Du Y. // Laser Photon. Rev. 2013. V. 7. N 5. P. 818-829.

[7] Wan Y., Inoue D., Jung D., Norman J.C., Shang C., Gossard A.C., Bowers J.E. // Photon. Res. 2018. V. 6. N 8. P. 776-781.

[8] Mintairov S.A., Kalyuzhnyy N.A., Lantratov V.M., Maximov M.V., Nadtochiy A.M., Rouvimov S., Zhukov A.E. // Nanotechnology. 2015. V. 26. N 38. P. 385202.

[9] Kryzhanovskaya N.V., Moiseev E.I., Zubov F.I., Mozharov A.M., Maximov M.V., Kalyuzhnyy N.A., Mintairov S.A., Guseva Yu.A., Kulagina M.M., Blokhin S.A., Berdnikov Yu., Zhukov A.E. // J. Appl. Phys. 2019. V. 126. N 6. P. 063107.

[10] Zubov F., Maximov M., Kryzhanovskaya N., Moiseev E., Muretova M., Mozharov A., Kaluzhnyy N., Mintairov S., Kulagina M., Ledentsov N., Jr., Chorchos L., Ledentstsov N., Zhukov A. // Opt. Lett. 2019. V. 44. N 22. P. 5442-5445.

[11] Зубов Ф.И., Моисеев Э.И., Корнышов Г.О., Крыжсановская Н.В., Шерняков Ю.М., Паюсов А.С., Кулагина М.М., Калюжсный Н.А., Минтаиров С.А., Максимов М.В., Жуков А.Е. // Письма в ЖТФ. 2019. Т. 45. В. 19. С. 37-39.

[12] Moiseev E., Kryzhanovskaya N., Maximov M., Zubov F., Nadtochiy A., Kulagina M., Zadiranov Yu., Kalyuzhnyy N., Mintairov S., Zhukov A. // Opt. Lett. 2018. V. 43. N 19. P. 4554-4557. 
[13] Жуков А.Е., Моисеев Э.И., Крыжсановская Н.В., Блохин С.А., Кулагина М.М., Гусева Ю.А., Минтаиров С.А., Калюжный Н.А., Можсаров А.М., Зубов Ф.И., Максимов М.В. // ФТП. 2019. Т. 53. В. 8. С. 1122-1127.

[14] Kryzhanovskaya N.V., Moiseev E.I., Zubov F.I., Mozharov A.M., Maximov M.V., Kalyuzhnyy N.A., Mintairov S.A., Kulagina M.M., Blokhin S.A., Kudryavtsev K.E., Yablonskiy A.N., Morozov S.V., Berdnikov Yu., Rouvimov S., Zhukov A.E. // Photon. Res. 2019. V. 7. N 6. P. 664-668.

[15] Slusher R.E., Levi A.F.J., Mohideen U., McCall S.L., Pearton S.J., Logan R.A. // Appl. Phys. Lett. 1993. V. 63. N 10. P. 1310-1313.

[16] Coldren L.A., Corzine S.W., Mašanović M.L. Diode lasers and photonic integrated circuits. 2nd ed. Hoboken, N.J.: John Wiley \& Sons, 2012. 709 p.

[17] Semiconductor lasers I. Fundamentals / Ed. E. Kapon. San Diego: Academic Press, 1999. 453 p. 